\title{
Decreased Event-Related Beta Synchronization During Memory Maintenance Marks Early Cognitive Decline in Mild Cognitive Impairment
}

\author{
Zsuzsanna Fodor ${ }^{\mathrm{a}}$, Enikő Sirály ${ }^{\mathrm{a}}$, András Horváth $^{\mathrm{b}}$, Pál Salacz ${ }^{\mathrm{a}, \mathrm{c}}$, Zoltán Hidasia $^{\mathrm{a}}$, \\ Éva Csibria ${ }^{\mathrm{a}}$, Ádám Szabód and Gábor Csukly ${ }^{\mathrm{a}, *}$ \\ ${ }^{a}$ Department of Psychiatry and Psychotherapy, Semmelweis University, Budapest, Hungary \\ ${ }^{\mathrm{b}}$ Department of Neurology, National Institute of Clinical Neurosciences, Budapest, Hungary \\ ${ }^{\mathrm{c}}$ Department of Neurology, Hospital at Péterfy Sándor Street, Budapest, Hungary \\ ${ }^{\mathrm{d}}$ MR Research Center, Semmelweis University, Budapest, Hungary
}

Handling Associate Editor: László Vécsei

Accepted 9 February 2018

\begin{abstract}
Mild cognitive impairment (MCI) refers to a measurable deficit in cognition in the absence of dementia or impairment in activities of daily living. Working memory impairment is among the earliest signs of MCI. Oscillatory analysis of working memory might be a potential tool for identifying patients at increased risk of developing dementia. Our study aimed to assess the temporospatial pattern of spectral differences during working memory maintenance between MCI patients and healthy controls and to compare the sources of oscillatory activity between the two groups. Event-related spectral perturbation of $17 \mathrm{MCI}$ patients and 21 healthy control participants was studied with 128-channel EEG during the Sternberg working memory task. Source localization was performed by using the eLORETA software. Among the participants, $13 \mathrm{MCI}$ and 15 control participants underwent a structural brain MRI examination. Event-related synchronization (ERS) in the alpha and beta frequency band was significantly lower in MCI patients compared to healthy control participants during retention. Both study groups showed significant memory load-related enhancement in both frequency band. In the MCI group, source localization revealed significantly attenuated beta oscillatory activity in the inferior and middle temporal gyrus, in the fusiform gyrus, and in the cuneus. Beta ERS correlated significantly with the size of the hippocampus, entorhinal cortex, and parahippocampal gyrus. During the retention period, MCI is characterized by decreased alpha and beta ERS compared to controls indicating early impairment in neural networks serving working memory maintenance. The assessment of electrophysiological changes in the beta frequency range may provide a useful diagnostic tool for the early detection of cognitive impairment.
\end{abstract}

Keywords: Alpha rhythm, beta rhythm, electroencephalography, memory, mild cognitive impairment, short-term

\section{INTRODUCTION}

Mild cognitive impairment (MCI) refers to a measurable deficit in cognition in the absence of dementia

\footnotetext{
*Correspondence to: Gábor Csukly, MD, PhD, 1083, Balassa utca 6, Budapest, Hungary. Tel.: +36 20825 0174; Fax: +36 1210 0336; E-mails: csukly.gabor@semmelweis-univ.hu and csugab@yahoo.com.
}

or impairment in activities of daily living [1]. MCI patients have an increased risk of developing dementia compared to the average elderly population. However, it could also be a stable or reverse condition without further progression [2]. Identifying patients with high risk of conversion has gained considerable interest, as these individuals are the best applicants for therapeutic intervention. 
This study is a continuation of our previously published works about structural and neuropsychological differences in MCI [3, 4], focusing on the spectral dynamics assessed with EEG and source localization of electrophysiological activity estimated by exact low-resolution brain electromagnetic tomography analysis (eLORETA) on the same population of subjects.

Working memory tasks are widely used paradigms in EEG studies of MCI [5-10], as working memory impairment is among the earliest signs of MCI [11-15] and it has been identified as a reliable predictor of Alzheimer's disease [11, 13, 16].

Previous analyses of event-related potentials (ERPs) were able to identify potential electrophysiological biomarkers of MCI [17]. Nevertheless, oscillatory analysis (event-related spectral perturbation, ERSP) brings important insights to memory deficits in MCI compared to conventional ERP analysis, since ERSP also captures the induced activity, which is not represented in ERPs. ERSP measures relative changes from the spectral power baseline, allowing the analysis of the time course of the EEG signal energy in specific frequency bands [18]. To gain a full insight into the electrophysiological activity linked to working memory, evoked and induced activity were analyzed by calculating the ERSP during the Sternberg working memory task.

Oscillatory activity in the alpha and beta frequency range plays an essential role in working memory. During the retention period of working memory tasks increased alpha [19-23] and beta power [24, 25] have been found consistently. Furthermore, memory load-related enhancement has been observed in both frequency bands [19, 20, 26, 27]. However, despite the similar dynamics, the two frequency bands might have different roles: alpha oscillation reflects a topdown mechanism of active suppression of irrelevant information $[19,22,28,29]$ and the top-down modulation of attention [21, 30], while beta oscillation is important in the active maintenance of new information for further task requirements [24, 26, 31].

In MCI, only a few studies examined alpha and beta event-related oscillatory activity during a working memory task. Decreased beta event-related synchronization (ERS) has been revealed in progressive MCI and Alzheimer's disease compared to stable MCI and healthy controls during a two-back task [8]. Decreased alpha/beta (10-20 Hz) activity was found in MCI compared to healthy controls in an auditory Sternberg memory task [7].
Most of the previous time-frequency analyses used the n-back task paradigm [5, 6, 8, 32]. The main advantage of the Sternberg task is the opportunity to separate the encoding, retention and retrieval conditions temporally. Therefore, we applied this paradigm in the present research. Furthermore, former studies did not investigate the effect of task difficulty on modulation of alpha and beta activity in MCI.

For the source localization, the low-resolution electromagnetic tomography approach was applied, which has been previously successfully used for the analysis of ERPs in MCI patients [33, 34]. However, to our knowledge, this is the first study examining the sources of induced time-varying oscillations in MCI.

Our study aimed to assess the temporospatial pattern of spectral differences between MCI patients and healthy controls in working memory processes as revealed by ERSP and to analyze the differences in the cortical projections of the time-varying crossspectral density between the two study groups. The main goal of this study was to find electrophysiological biomarkers that mark deteriorated retention in MCI.

Based on the previous research, we assumed a modulation of alpha and beta oscillations by memory load, and decreased alpha and beta ERS of the temporal lobe in MCI patients compared to controls, reflecting the early impairment of working memory network. Furthermore, we hypothesized a correlation between alpha and beta ERS and other early markers of cognitive decline such as neuropsychological tests and the volume and cortical thickness of medial temporal lobe structures measured by MRI.

\section{MATERIALS AND METHODS}

\section{Participants and clinical measures}

The study was carried out in the Department of Psychiatry and Psychotherapy, Semmelweis University, Budapest, Hungary. Altogether 17 MCI patients and 21 healthy control participants were enrolled in the study. Among them, 13 MCI patients and 15 healthy control participants underwent a structural brain MRI examination (others refused participation). Demographic and neuropsychological data are summarized in Table 1.

Selection criteria were no history of head injury, stroke, mental retardation, epileptic seizure, acute psychiatric disorder or substance dependence. Subjects with dementia were excluded according to 
Table 1

Demographic data and results of basic neuropsychological tests

\begin{tabular}{|c|c|c|c|}
\hline & control $(n=21)$ & $\operatorname{MCI}(n=17)$ & $p$ value \\
\hline Age (Mean (SD)) & $64.9(5.2)$ & $69.2(7)$ & $p=0.04$ \\
\hline Education $^{\mathrm{a}}$ & $19 \% / 19 \% / 62 \%$ & $18 \% / 18 \% / 65 \%$ & n.s.* \\
\hline Gender (Female) & $66,70 \%$ & $47 \%$ & n.s. $*$ \\
\hline RAVLT $1-5$ sum $^{\mathrm{b}}$ & $54.3(6.4)$ & $40.4(10.8)$ & $p<0.0001$ \\
\hline RAVLT delayed recallc (MCI: $n=14$ ) & $10.9(2.4)$ & $7(4.3)$ & $p=0.002$ \\
\hline ACE Total Score ${ }^{\mathrm{d}}$ & $94.5(2.9)$ & $87(7.7)$ & $p=0.0002$ \\
\hline ACE VL/OM-ratio ${ }^{\mathrm{e}}$ & $2.6(0.4)$ & $2.7(0.5)$ & n.s. $*$ \\
\hline MMSE Total Score ${ }^{f}$ & $29(1.2)$ & $28.1(1.2)$ & $p=0.04$ \\
\hline TMT Part $A^{g}$ & $34.4(8.5)$ & $70.2(52.8)$ & $p<0.0001$ \\
\hline TMT Part B $($ MCI: $n=16)$ & $63.1(16)$ & $142.5(68.4)$ & $p<0.0001$ \\
\hline GDS Score $^{\mathrm{h}}$ (control: $n=20$ ) & $4.4(3.2)$ & $4.2(3.5)$ & n.s.* \\
\hline STAI Score ${ }^{\mathrm{i}}$ & $40.8(11.4)$ & $35.9(9.4)$ & n.s.* \\
\hline Sternberg test ${ }^{\mathrm{j}}$ & $91.7 \%(8.5)$ & $82.3 \%(19.7)$ & $p=0.049$ \\
\hline
\end{tabular}

MCI, mild cognitive impairment, ACE, Addenbrooke's Cognitive Examination; STAI, State-Trait Anxiety Inventory; MMSE, Mini-Mental State Examination; GDS, Geriatric Depression Scale; RAVLT, Rey Auditory Verbal Learning Test; TMT, Trail Making Test. ${ }^{a}$ Participants were categorized into three education groups: $1=$ less than 12 years; 2 = high school graduation (12 years education); $3=$ more than 12 years of education. ${ }^{\mathrm{b}}$ Sum of all words in the first five trials. The maximum score is $75 .{ }^{\mathrm{c}}$ The maximum score is 15 . $^{\mathrm{d}}$ The maximum score is $100 .{ }^{\mathrm{e}} \mathrm{VL} / \mathrm{OM}$ : verbal fluency and language points/orientation and delayed recall ratio can be defined based on ACE. Result below 2.2 indicate frontotemporal dementia and result over 3.2 indicate Alzheimer's disease. ${ }^{\mathrm{f}}$ The maximum score is 30. ${ }^{\mathrm{g}}$ Time needed for completing the task in seconds. ${ }^{\mathrm{h}}$ The maximum score is 15 . ${ }^{\mathrm{i}}$ The maximum score is 80 . ${ }^{\mathrm{j}}$ Response accuracy in the Sternberg task. *n.s. (not significant) $=p>0.05$.

the Mini-Mental State Examination (MMSE) scores standardized for age and education [35].

Participants had subjective memory complaints and applied to take part in a cognitive training program announced among general practitioners and in a Retirement Home (The study is registered at ClinicalTrials.gov, the identifier is 'NCT02310620'). Every participant underwent a regular psychiatric assessment to evaluate possible excluding comorbidity. After that, cognitive functions were assessed with neuropsychological tests to specify the diagnosis.

Diagnostic procedure of MCI was based on the Petersen criteria [1], including subjective memory complaint corroborated by an informant, preserved everyday activities, memory impairment based on a standard neuropsychological test, preserved global cognitive functions and the exclusion of dementia. For the detailed assessment of memory impairment, we applied the Rey Auditory Verbal Learning Test (RAVLT). Attention, executive functions, and cognitive flexibility were examined with the Trail Making Test (TMT) Part A and Part B, global cognitive performance was estimated with the Addenbrooke's Cognitive Examination (ACE). For the differentiation between MCI and healthy controls, we applied a cutoff score of $1 \mathrm{SD}$ under population mean standardized for age and gender/education in these neuropsychological tests. Participants, who scored under the cut-off value in the delayed recall subscore or the total score of RAVLT or the TMT Part B or the ACE, were put into the MCI group. The results of the neuropsychological tests are summarized in Table 1.

\section{EEG stimuli and procedures}

During EEG examination, participants were seated in a dimly lit, sound-attenuated room. White digits, Arial font, point size 60 were presented as stimuli on a computer screen at approximately $50 \mathrm{~cm}$ with Presentation 13.0 software (Neurobehavioral Systems, Inc.; Albany, CA). All participants had normal or corrected-to-normal vision.

During EEG recording subjects performed the Sternberg task [36], an extensively used test of working memory $[7,22]$.

At the onset of each trial, three, four or five randomly selected one-digit number appeared sequentially in the middle of the screen for $2000 \mathrm{~ms}$, separated by a fixation cross for $450-500 \mathrm{~ms}$. The length of the sequence was counterbalanced across 126 trials. An exclamation mark showed for $500 \mathrm{~ms}$ indicated the end of the learning sequence. For the retention period, a fixation cross appeared for $3900-4000 \mathrm{~ms}$. In the retrieval condition, two probe numbers were presented for $2000 \mathrm{~ms}$ separated by fixation cross presented for $450-500 \mathrm{~ms}$ (Fig. 1). 


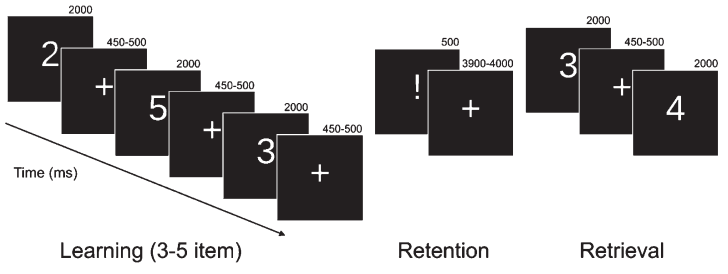

Fig. 1. The Sternberg working memory task.

Participants were instructed to indicate by clicking with the mouse (yes-right/no-left) whether the probe number was part of the presented sequence. Response assignment was counterbalanced across trials. Efficiency was measured by response accuracy.

We ascertained that the participants are alert and understand the instructions, and we monitored them during the completion of the task to bypass the possible distorting effect of extended eye closure on the EEG activity, especially in the alpha frequency range [37]. For the same purpose, participants completed the task in six equal parts separated by a 3-min rest period.

\section{EEG recording and processing}

EEG was recorded from DC with a low-pass filter at $100 \mathrm{~Hz}$ using a high-density 128-channel BioSemi ActiveTwo amplifier [38]. Electrode caps had an equidistant-layout and covered the whole head. Eye movements were monitored with EOG electrodes placed below the left and above the right external canthi. Data were digitized at a sampling rate of $1024 \mathrm{~Hz}$. Built-in and self-developed functions as well as the freeware EEGLAB toolbox [39] in the Matlab (MathWorks, Natick, MA) development environment was used for subsequent off-line data analyses. EEG was re-referenced to the common average potential and filtered off-line between 0.5 and $45 \mathrm{~Hz}$ using zerophase shift forward, and reverse IIR Butterworth filter.

Epochs from $500 \mathrm{~ms}$ pre-stimulus to $2000 \mathrm{~ms}$ post-stimulus for the learning and $3500 \mathrm{~ms}$ poststimulus for the retention period were extracted from the continuous EEG and corrected for the pre-stimulus baseline. Removal of muscle, blinking, and eye movement artifacts (detected by EOG) were performed by ADJUST [40], an ICA (Independent Component Analysis) based automatic artifact detector. Furthermore, epochs with a voltage exceeding $\pm 100 \mu \mathrm{V}$ on any channel were rejected from the analysis.
After artifact rejection, the average number of trials in the control group and MCI group were 478.1 $(\mathrm{SD}=40.2), 414.6(\mathrm{SD}=66.8)$ for the learning condition and 119.4 $(\mathrm{SD}=13.3), 103.8(\mathrm{SD}=19.2)$ for the retention condition, respectively

We divided the 128 channels into 11 scalp regions (three frontal, three central, three parieto-occipital and two temporal).

\section{EEG data analysis}

Stimulus-related alpha $(7-14 \mathrm{~Hz})$ and beta $(14-25 \mathrm{~Hz})$ activity changes were measured by the ERSP, providing a 2-D representation of the mean change in spectral power (in $\mathrm{dB}$ ) from baseline [41]. Beyond conventional event-related analytical approach, ERSP is able to capture event-related as well as induced oscillatory activity. Unlike evoked responses, induced activity is not phase-locked to the stimulus, and if a brain response to an event is not phase-locked across trials precisely enough, the potentially important induced activity will be averaged out from the ERPs, and therefore poorly represented in, or completely absent from the time-domain features of the ERP.

To compute the ERSP, baseline spectra are calculated from the EEG immediately preceding each event. The epoch is divided into brief, overlapping data windows, and a moving average of the amplitude spectra is created. Each of these spectral transforms of individual response epochs is then normalized by dividing by their respective mean baseline spectra. Normalized response transforms for many trials are then averaged to produce the average ERSP, plotted as relative spectral log amplitude on a time-by-frequency plane [41] (see details in the Supplementary Material).

The analysis was performed on epochs extending from $500 \mathrm{~ms}$ before to $2000 \mathrm{~ms}$ and $3500 \mathrm{~ms}$ after stimulus onset in learning and retention condition, respectively in the $1.5-30 \mathrm{~Hz}$ frequency range. The sliding window was $400 \mathrm{~ms}$ wide, and it was applied 200 times with an average step size of $6 \mathrm{~ms}$. No zero padding was applied. The analyzed time interval lasted from $300 \mathrm{~ms}$ before to $1800 \mathrm{~ms}$ and 3300 after stimulus onset in learning and retention condition, respectively. The ERSP time-frequency matrices were baseline corrected by the average power calculated from the 500 to $200 \mathrm{~ms}$ pre-stimulus period. Dynamical changes in oscillatory activity were studied by computing ERSPs for each trial, then averaging them separately for learning and retention condition 
$[42,43]$. Mean ERSP values were calculated by averaging across electrodes within scalp regions to further attenuate noise.

Based on a prior study [8] and adjusted to the end of desynchronization, we selected the $800-1500 \mathrm{~ms}$ (alpha band) and the 800-1700 ms (beta band) time windows in learning condition for further analysis. Epochs of retention were sorted into low and high memory load condition, according to the length of the learning sequence (three-item vs. four and five-item), to assess memory load-related spectral modulation. In retention condition, the analysis was performed on the 600-1000 ms time window (alpha and beta band).

\section{Source localization}

Based on the scalp-recorded electric potential distribution, the exact low-resolution brain electromagnetic tomography (eLORETA) software (free academic software at http://www.uzh.ch/keyinst/loreta. $\mathrm{htm})$ was used to compute the cortical threedimensional distribution of current density. The eLORETA method is a discrete, three-dimensional (3D) distributed, linear, weighted minimum norm inverse solution, where the intracerebral volume is partitioned in 6239 voxels at $5 \mathrm{~mm}$ spatial resolution. Description of the method together with the proof of its exact zero-error localization property, are in $[44,45]$.

For source localization, the artifact-corrected EEG was down-sampled to $256 \mathrm{~Hz}$. Time-varying crossspectra of epochs were calculated using a Hamming window of $400 \mathrm{~ms}$ width. Subsequently, current source density of alpha and beta oscillations was estimated in eLORETA for voxels as a function of time.

\section{MR image acquisition and processing}

Participants underwent a routine brain MR examination, producing high-resolution anatomical images used for analysis. Image acquisitions were made at the MR Research Center, Semmelweis University, Budapest on a 3 Tesla Philips Achieva clinical MRI scanner equipped with an 8-channel SENSE headcoil. High resolution, whole brain anatomical images were obtained using a $\mathrm{T} 1$ weighted 3-dimensional spoiled gradient echo (T1W 3D TFE) sequence (see details in the Supplementary Material).

\section{Statistical analysis}

The different effects on ERSP in learning condition were tested by two-way analyses of covariance (ANCOVA) of the study group (HC versus $\mathrm{MCI}) \times$ region. To test the effect of memory load on ERSP three-way ANCOVA of study group $\times$ region $\times$ memory load (three- vs. four and five-item) was applied for the analysis in retention period. All the main effects including age and gender, two- way and three-way interactions for the second model were included into the ANCOVA models.

Post-hoc pairwise contrasts were conducted to investigate the interactions. Since between-group comparisons were evaluated over eleven regions, Hochberg correction for multiple comparisons was applied to the post-hoc contrasts [46, 47].

Group differences were characterized by Cohen's d. Associations between neuropsychological test scores, size of medial temporal lobe structures and ERSP in regions of large [48] between-group difference in learning (Cohen's $d>0.8$ ) and regions of large load-related difference in retention condition (Cohen's d $>1.0$ ) were assessed. For the analysis, Pearson correlation was applied adjusted for age. For variables, deviating largely from the normal distribution Spearman correlation was used adjusted for age. Structural MRI results were derived from previous, former published part of our study, as the size of medial temporal lobe structures could most effectively distinguish MCI patients from healthy controls $[3,4]$.

In eLORETA, voxel-by-voxel comparisons were estimated for learning condition and retention period for the previously defined time windows between the subject-wise corrected control and MCI groups by using independent $t$-test (Baseline Corrected Control vs. Baseline Corrected MCI). Statistical significance was assessed with a nonparametric randomization test $(n=5000)$ that corrects for multiple comparisons [49].

\section{RESULTS}

\section{Behavioral results}

The MCI group (mean score $=82.3 \%, \mathrm{SD}=19.7$ ) showed a significantly decreased memory performance ( $\mathrm{U}=111, p=0.049)$ compared to the control group (mean score $=91.7 \%, \mathrm{SD}=8.5$ ) in the Sternberg task. 



(B)
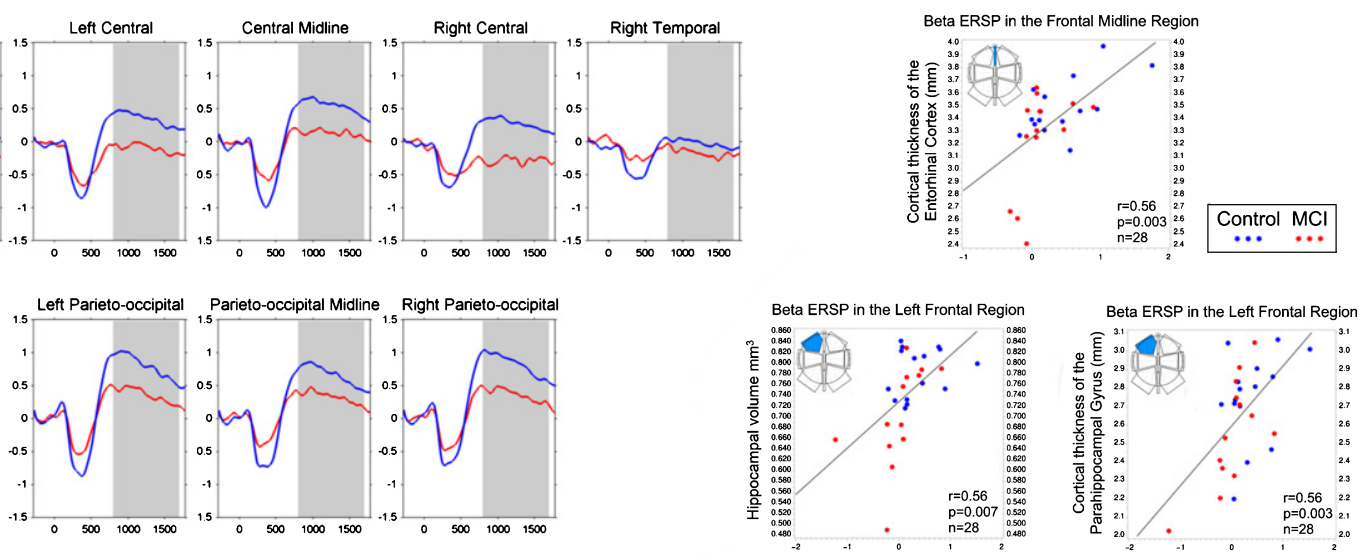

(A)

(C)

Fig. 2. Late learning/early retention condition. A) Beta event-related spectral perturbation (ERSP) in study groups (800-1700 ms timewindow highlighted). B) Scalp topography of the beta ERSP in study groups in the 800-1700 ms time window. C) Correlation of beta ERSP with the size of medial temporal lobe structures in the 800-1700 ms time window.

\section{Alpha synchronization in late learning/early retention}

During learning condition in the $800-1500 \mathrm{~ms}$ time window control subjects showed a significant eventrelated synchronization (ERS) in the alpha frequency band in all scalp regions (i.e., differences from zero were significant at a corrected level of $p<0.05)$, while in the MCI group alpha no similar ERS was observable (corrected $p>0.05$ ).

A significantly decreased alpha ERS $(\mathrm{F}(1,34)=4.44, \quad p=0.04)$ was detected in the MCI group relative to controls. The main effect of age and gender on alpha ERSP were not significant $(p>0.05)$, while the interaction of region and group had a significant effect $(\mathrm{F}(10,34)=4.75, p=0.0003)$. Post hoc analyses of this interaction revealed large between-group differences in the right central and left temporal regions (Cohen's $d=1.1$ and 0.9). The between-group difference in the right central region $(t=3.47, \mathrm{df}=34, p=0.001)$ remained significant after correction for multiple comparisons.

\section{Beta synchronization in late learning/early retention}

Control subjects showed significant beta ERS in all frontal, midline and parieto-occipital scalp regions in the late $800-1700 \mathrm{~ms}$ time window for learning condition. While in the MCI group beta ERS was only observable in the left parieto-occipital and midline parieto-occipital regions in the same time window (corrected $p<0.05$ ) (Fig. 2A, B).

A significantly decreased beta ERS $(\mathrm{F}(1,34)=6.69, p=0.01)$ was detected in the MCI group relative to the control group. The main effect of age and gender were not significant $(p>0.05)$, while region and group had a significant interaction effect $(F(10,34)=3.13, p=0.006)$. Between-group differences were large in terms of Cohen's $d$ in midline (frontal: 0.9 , central: 1.0 , parieto-occipital: 0.9), pariteo-occipital (left: 1.0, right: 0.9) and left frontal (0.9) regions. The between-group difference in the central midline region $(t=3.04, \mathrm{df}=34$, $p=0.0045)$ remained significant after correction for multiple comparisons.

\section{Correlational analysis of alpha and beta synchronization in late learning/early retention}

Significant negative correlations were found between the time needed for participants to complete TMT Part A and Part B and alpha ERS in right central (A: Spearman $r=-0.49, p=0.002$, B: Spearman $r=-0.52, p=0.001)$ and left temporal regions 
(A: Spearman $r=-0.45, p=0.005$. B: Spearman $r=-0.50, p=0.002)$. Alpha ERS did not correlate significantly with the size of medial temporal lobe structures.

Beta ERS in early retention showed significant correlations with the ACE total score, TMT Part A, and Part B results, RAVLT delayed recall and with response accuracy in the Sternberg task. Additionally, beta ERS correlated significantly with the cortical thickness of the entorhinal cortex and parahippocampal gyrus and the volume of the hippocampus (Fig. 2C, Table 2).

\section{Source localization of alpha and beta synchronization in late learning/early retention}

Group comparison revealed a significantly (corrected non-parametric $p<0.05$ ) lower beta activity in MCI group in temporal, occipital and frontal lobe relative to controls. In the beta band, the most expanded clusters with voxels of significant between-group differences were localized in inferior temporal gyrus, middle temporal gyrus, and fusiform gyrus (Fig. 3, Table 3).

In the alpha frequency band, tendency level (corrected non-parametric $p<0.1$ ) differences were observed between groups in frontal and parietal lobe structures.

\section{Alpha and beta synchronization in late retention with varying memory load}

During retention, in the $600-1000 \mathrm{~ms}$ time window, both groups showed increased alpha activity in high memory load (four- and five-item learning sequence) condition compared to low memory load (three-item learning sequence) $(\mathrm{F}(1,34)=42.9$, $p<0.0001)$. However, the alpha band did not show significant between-group difference.

Contrary to that in beta frequency band significantly lower beta ERS $(\mathrm{F}(1,34)=6.26, p=0.02)$ was observable in MCI participants relative to controls (Fig. 4A, B). Beta ERS increased significantly with memory load $(\mathrm{F}(1,34)=45.97, p<0.0001)$ in both groups similarly, as the three-way interaction of group, load, and the region was not significant $(p>0.05)$.

The modulation in the beta band was large (Cohen's $d>0.8$ ) in all regions and reached its maximum in the right central region (Cohen's $\mathrm{d}=1.2$ ). Interestingly, this region showed the largest between-group differences both in low and high memory load conditions (Cohen's $\mathrm{d}=0.94$ and 0.73$)$.

\section{Correlational analysis of beta synchronization in late retention}

Correlational analysis was performed for the beta frequency range, as alpha activity did not show significant between-group difference.

Mean beta ERS of late retention condition correlated significantly with the RAVLT immediate recall and the TMT Part A and Part B scores. Furthermore, beta ERS showed a significant correlation with the cortical thickness of the entorhinal cortex and the volume of the hippocampus (Fih. 4C, Table 2).

\section{Source localization of beta synchronization in late retention}

A tendency level (corrected non-parametric $p<0.1)$ between-group difference was observed in the beta activity of the precentral gyrus (Table 3 ).

\section{DISCUSSION}

In this study, the electrophysiological correlates of working memory maintenance were examined in MCI patients and healthy controls.

Retention of information is deteriorated in MCI [50] and neuropsychological tests, assessing the efficacy of the maintenance of newly learned information are considered as sensitive instruments of early detection of MCI [51, 52].

Previous studies found that alpha and beta oscillations are linked to working memory maintenance $[20,22]$. Therefore we specifically examined changes in these frequency bands applying the neural synchrony approach. For the assessment, we used the Sternberg memory paradigm allowing temporal separation of encoding, retention, and retrieval.

During the learning condition, various cognitive processes can be distinguished: encoding of new stimulus, adding to the pool of memory contents and maintenance of the newly acquired information. A late time window was applied similarly to a previous study [8] assuming, that oscillatory changes during this late time window reflect neural activity related to the early period of retention rather than encoding. Our assumption is corroborated by the observed dominance of alpha and beta synchronization during this time window $[19,24,31]$ in contrast 
Table 2

Correlations between the neuropsychological test results, temporal lobe structures and the Beta Event-Related Synchronization (ERS) across the entire sample

\begin{tabular}{|c|c|c|c|c|c|c|c|c|c|c|}
\hline & \multicolumn{10}{|c|}{ Beta ERS } \\
\hline & \multicolumn{5}{|c|}{ Early retention } & \multicolumn{5}{|c|}{ Late retention } \\
\hline & Midline & $\begin{array}{c}\text { Left } \\
\text { Parieto- } \\
\text { occipital } \\
\end{array}$ & $\begin{array}{l}\text { Frontal } \\
\text { Midline }\end{array}$ & $\begin{array}{c}\text { Right } \\
\text { Parieto- } \\
\text { occipital } \\
\end{array}$ & Left Frontal & $\begin{array}{l}\text { Parieto- } \\
\text { occipital } \\
\text { Midline } \\
\end{array}$ & $\begin{array}{l}\text { Right } \\
\text { Central }\end{array}$ & $\begin{array}{c}\text { Left } \\
\text { Central }\end{array}$ & $\begin{array}{l}\text { Right } \\
\text { Frontal }\end{array}$ & $\begin{array}{c}\text { Right } \\
\text { Temporal } \\
\end{array}$ \\
\hline \multicolumn{11}{|l|}{ Neuropsychological test } \\
\hline $\begin{array}{l}\text { ACE Total Score }(n=38) \\
\text { Spearman } r(p \text {-value })\end{array}$ & $0.32(0.06)$ & $0.29(0.09)$ & $0.18(0.3)$ & $0.26(0.1)$ & $0.32(0.05)$ & $0.36(0.03)$ & $0.18(0.3)$ & $0.12(0.5)$ & $0.12(0.5)$ & $0.27(0.1)$ \\
\hline $\begin{array}{l}\text { RAVLT items } 1-5 \text { sum } \\
\quad(n=38) \text { Pearson } r \\
\text { (p-value })\end{array}$ & $0.27(0.1)$ & $0.17(0.3)$ & $0.16(0.3)$ & $0.13(0.4)$ & $0.21(0.2)$ & $0.19(0.3)$ & $0.35(0.03)$ & $0.07(0.7)$ & $0.22(0.2)$ & $0.26(0.1)$ \\
\hline $\begin{array}{l}\text { RAVLT delayed recall } \\
\quad(n=35) \text { Pearson } r \\
\text { (p-value) }\end{array}$ & $0.39(0.02)$ & $0.15(0.4)$ & $0.23(0.2)$ & $0.13(0.5)$ & $0.14(0.4)$ & $0.23(0.2)$ & $0.25(0.1)$ & $-0.004(1.0)$ & $0.16(0.4)$ & $0.26(0.1)$ \\
\hline $\begin{array}{l}\text { TMT part A }(n=38) \\
\text { Spearman } r(p \text {-value })\end{array}$ & $-0.55(0.0004)$ & $-0.42(0.01)$ & $-0.30(0.07)$ & $-0.47(0.003)$ & $-0.41(0.01)$ & $-0.37(0.03)$ & $-0.37(0.02)$ & $-0.30(0.07)$ & $-0.22(0.2)$ & $-0.25(0.1)$ \\
\hline $\begin{array}{l}\text { TMT part B }(n=37) \\
\quad \text { Spearman } r(p \text {-value })\end{array}$ & $-0.53(0.0008)$ & $-0.47(0.004)$ & $-0.38(0.02)$ & $-0.45(0.006)$ & $-0.48(0.003)$ & $-0.49(0.002)$ & $-0.38(0.02)$ & $-0.21(0.2)$ & $-0.32(0.06)$ & $-0.21(0.2)$ \\
\hline $\begin{array}{l}\text { Sternberg task }(n=38) \\
\text { Spearman } r(p \text {-value })\end{array}$ & $0.27(0.1)$ & $0.25(0.1)$ & $0.10(0.54)$ & $0.34(0.04)$ & $0.21(0.2)$ & $0.33(0.0495)$ & $-0.01(0.9)$ & $-0.12(0.5)$ & $0.16(0.4)$ & $0.17(0.3)$ \\
\hline \multicolumn{11}{|l|}{ Cortical thickness (mm) } \\
\hline $\begin{array}{l}\text { Entorhinal cortex }(n=28) \\
\quad \text { Pearson } r(p \text {-value })\end{array}$ & $0.45(0.02)$ & $0.46(0.02)$ & $0.56(0.003)$ & $0.4(0.04)$ & $0.47(0.01)$ & $0.46(0.02)$ & $0.55(0.003)$ & $0.54(0.004)$ & $0.46(0.02)$ & $0.45(0.02)$ \\
\hline $\begin{array}{l}\text { Parahippocampal gyrus } \\
(n=28) \text { Pearson } r \\
(p \text {-value }) \\
\end{array}$ & $0.48(0.01)$ & $0.55(0.003)$ & $0.39(0.04)$ & $0.46(0.02)$ & $0.56(0.003)$ & $0.41(0.03)$ & $0.18(0.4)$ & $0.06(0.8)$ & $0.10(0.6)$ & $0.09(0.6)$ \\
\hline \multicolumn{11}{|l|}{ Volume $\left(\mathrm{mm}^{3}\right)$} \\
\hline $\begin{array}{l}\text { Hippocampus }(n=28) \\
\text { Pearson } r(p \text {-value })\end{array}$ & $0.32(0.1)$ & $0.44(0.02)$ & $0.37(0.06)$ & $0.46(0.02)$ & $0.51(0.007)$ & $0.40(0.04)$ & $0.40(0.04)$ & $0.39(0.046)$ & $0.26(0.2)$ & $0.39(0.45)$ \\
\hline
\end{tabular}




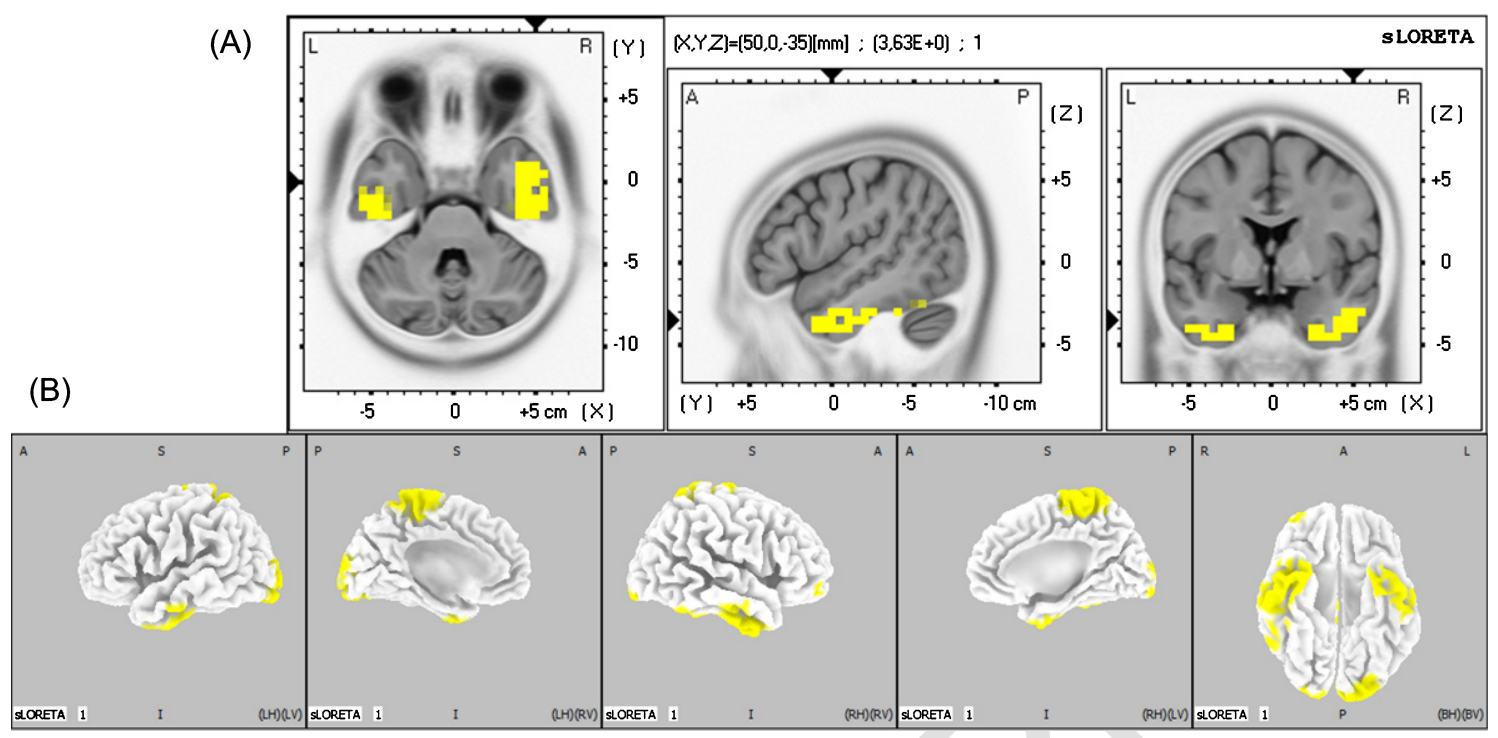

Fig. 3. Source localization of beta oscillatory activity in late learning/early retention condition. A) Largest cluster with voxels of significant $(p<0.05)$ between-group difference in the inferior temporal gyrus. B) The result of the source localization in late learning/early retention condition. Highlighting indicates significant $(p<0.05)$ group differences in beta activity (Control $>\mathrm{MCI})$ in the $800-1700 \mathrm{~ms}$ time window.

to desynchronization, which characterizes encoding [53]. Therefore, we refer to this late time window as an early period of retention.

\section{Beta frequency band}

During early and late retention beta ERS in MCI group appeared only in the posterior regions, while the control group showed significant beta ERS in almost all scalp regions.

Converging evidence suggests that beta oscillation is an essential contributor for the sufficient functioning of working memory. Previous studies found beta activity enhancement during the retention period in various memory tasks [24, 25], that has been suggested to reflect the process of active maintenance of newly acquired information for further task requirements [8, 24, 26, 31].

We observed diminished beta ERS during early and late retention in the MCI group relative to controls, which is consistent with the results of previous studies $[7,8]$.

Significant correlation of beta ERS with response accuracy in Sternberg task and TMT results further supports the role of beta oscillations in active maintenance of information, as TMT Part B specifically reflects the ability to maintain two tracks of thought simultaneously [35].

The active role of beta oscillations is also corroborated by its observed enhancement according to increasing task demands in line with previous results of healthy individuals $[20,26]$. Our results show that in $\mathrm{MCI}$ this adaptive modulation is preserved.

While the differences of beta ERS between the two groups were significant in both condition, the reduction of beta oscillation in the MCI group did not have a region-specific distribution, indicated by similar effect sizes across scalp regions. In order to gain insight into the source of diminished beta activity in MCI, we used eLORETA for source localization. The results showed that the decreased beta ERS in the MCI group during early retention is linked to attenuated beta activity in the inferior and middle temporal gyrus, in the fusiform gyrus, and in the cuneus. These cortical areas play an important role in processing and encoding visual information. Our finding fit to the framework suggesting that sustained activity of the cortical regions involved in processing the representations of information may contribute to the neural process of maintenance in working memory $[54,55]$. Attenuated beta activity in task-related areas in the MCI group might reflect the disturbance of the cortical network supporting retention of information during memory task.

Furthermore, beta ERS during early and late retention showed significant positive correlation with the volume of the entorhinal cortex, the parahippocampal gyrus, and the hippocampus. Working memory maintenance stands on the complementary functions of the prefrontal cortex, medial temporal lobe and 
Table 3

Results of the source localization by eLORETA

\begin{tabular}{|c|c|c|c|c|c|c|c|c|c|}
\hline \multirow[t]{2}{*}{ Condition } & \multirow[t]{2}{*}{$\begin{array}{l}\text { Frequency } \\
\text { band }\end{array}$} & \multirow[t]{2}{*}{ Time-window } & \multirow[t]{2}{*}{ Areas } & \multirow[t]{2}{*}{$\begin{array}{l}\text { Brodmann } \\
\text { Areas }\end{array}$} & \multicolumn{3}{|c|}{$\begin{array}{l}\text { MNI coordinates } \\
\text { at } t_{\max }\end{array}$} & \multirow{2}{*}{$\begin{array}{l}\text { cluster } \\
\text { size } \\
\text { (voxel) }\end{array}$} & \multirow{2}{*}{$\begin{array}{l}\text { volume } \\
\left(\mathrm{cm}^{3}\right)\end{array}$} \\
\hline & & & & & $X$ & $Y$ & $Z$ & & \\
\hline \multirow[t]{24}{*}{ Early retention } & Alpha & $800-1500 \mathrm{~ms}$ & Postcentral Gyrus(*) & $3,4,5,7$ & -20 & -35 & 70 & 24 & 3 \\
\hline & & & $\begin{array}{l}\text { Medial Frontal } \\
\text { Gyrus }(*)\end{array}$ & 6 & -10 & -15 & 70 & 21 & 2.625 \\
\hline & & & Precuneus(*) & 7 & -5 & -65 & 60 & 19 & 2.375 \\
\hline & & & Paracentral Lobule $(*)$ & $4,5,6$ & 10 & -50 & 65 & 13 & 1.625 \\
\hline & & & Precentral Gyrus(*) & 4,6 & -15 & -30 & 70 & 11 & 1.375 \\
\hline & & & $\begin{array}{l}\text { Superior Frontal } \\
\text { Gyrus }(*)\end{array}$ & 6 & -15 & -15 & 70 & 8 & 1 \\
\hline & & & $\begin{array}{l}\text { Superior Parietal } \\
\text { Lobule }(*)\end{array}$ & 7 & 15 & -60 & 65 & 7 & 0.875 \\
\hline & Beta & $800-1700 \mathrm{~ms}$ & $\begin{array}{l}\text { Inferior Temporal } \\
\text { Gyrus* }\end{array}$ & $20,21,37$ & 50 & 0 & -35 & 71 & 8.875 \\
\hline & & & $\begin{array}{l}\text { Middle Temporal } \\
\text { Gyrus* }\end{array}$ & 21,38 & 50 & 10 & -40 & 33 & 4.125 \\
\hline & & & Fusiform Gyrus* & $18,19,20,36,37$ & -25 & -85 & -20 & 31 & 3.875 \\
\hline & & & Cuneus* & $17,18,19$ & -25 & -95 & -5 & 29 & 3.625 \\
\hline & & & Postcentral Gyrus* & $3,4,5,7$ & 10 & -35 & 70 & 29 & 3.625 \\
\hline & & & Paracentral Lobule** & $4,5,6$ & 0 & -35 & 65 & 24 & 3 \\
\hline & & & $\begin{array}{l}\text { Medial Frontal } \\
\text { Gyrus** }\end{array}$ & 6 & 5 & -30 & 70 & 21 & 2.625 \\
\hline & & & Uncus* & $20,36,38$ & 30 & 0 & -45 & 21 & 2.625 \\
\hline & & & $\begin{array}{l}\text { Middle Occipital } \\
\text { Gyrus* }\end{array}$ & 18,19 & -25 & -85 & -15 & 16 & 2 \\
\hline & & & Lingual Gyrus** & 17,18 & -25 & -95 & -10 & 12 & 1.5 \\
\hline & & & $\begin{array}{l}\text { Superior Temporal } \\
\text { Gyrus* }\end{array}$ & 38 & 35 & 15 & -45 & 9 & 1.125 \\
\hline & & & $\begin{array}{l}\text { Inferior Occipital } \\
\text { Gyrus** }\end{array}$ & 17,18 & -25 & -90 & -15 & 8 & 1 \\
\hline & & & Precentral Gyrus** & 4,6 & 20 & -20 & 70 & 7 & 0.875 \\
\hline & & & $\begin{array}{l}\text { Superior Frontal } \\
\text { Gyrus** }\end{array}$ & 6 & 10 & -20 & 70 & 7 & 0.875 \\
\hline & & & $\begin{array}{l}\text { Middle Frontal } \\
\text { Gyrus* }\end{array}$ & 10,11 & 45 & 55 & -5 & 4 & 0.5 \\
\hline & & & $\begin{array}{l}\text { Superior Parietal } \\
\text { Lobule* }\end{array}$ & 7 & -25 & -60 & 65 & 3 & 0.375 \\
\hline & & & Temporopolar Area* & 38 & 25 & 10 & -45 & 1 & 0.125 \\
\hline Late retention & Beta & $600-1000 \mathrm{~ms}$ & Precentral Gyrus(*) & 6 & 20 & -20 & 70 & 2 & 0.25 \\
\hline
\end{tabular}

$\left.{ }^{*}\right) p<0.1$, two-tailed, $* p<0.05$, two-tailed, ${ }^{*} p<0.01$, two-tailed.

cortical regions responsible for processing the representations [56]. In this network, the medial temporal lobe modulates the activity of task-specific posterior areas [57]. The beta oscillatory activity might be a candidate for mediating this connection, as memory load-related enhancement of beta phase synchronization was observed between the medial temporal lobe and the inferior temporal cortex [58]. Therefore, decreased beta activity of task-relevant cortical regions in MCI might reflect deteriorated regulatory function of the medial temporal lobe and might be an indirect marker of subtle functional impairment.

Our results complement previous studies referring decreased beta ERD during encoding [59] and recognition [7, 60]. and decreased beta ERS during retention [8] in MCI, leading us to the conclusion that reduced beta reactivity in working memory tasks could be a sensitive marker of early, subtle cognitive deterioration $[7,61]$.

\section{Alpha frequency band}

In line with the result of former studies we observed memory load-related enhancement of alpha activity $[22,23,27]$ in both study groups and decreased alpha ERS in the MCI group relative to controls during early retention [7].

Oscillatory changes in the alpha frequency range are often explained in the context of the active inhi- 


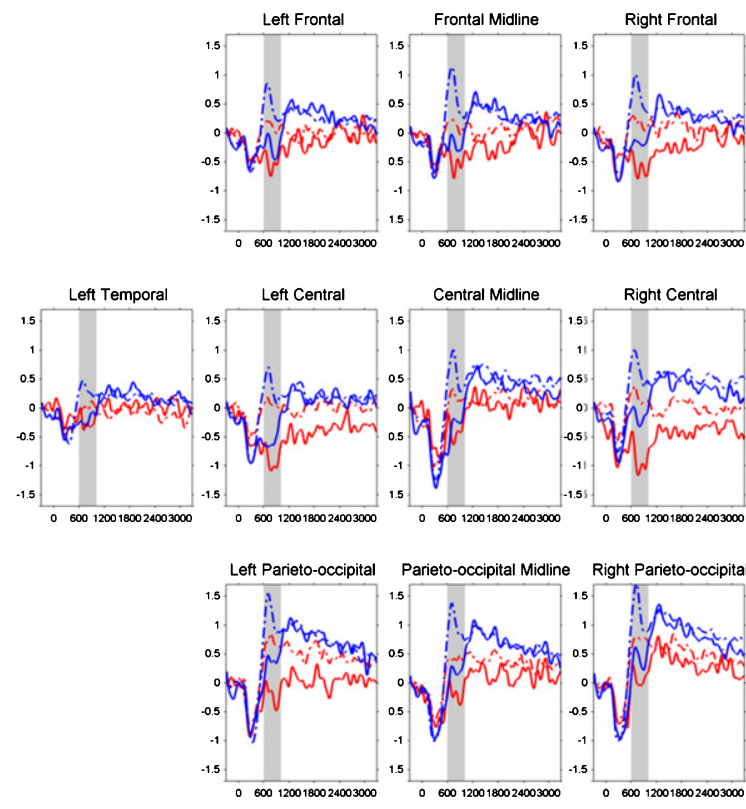

(A)

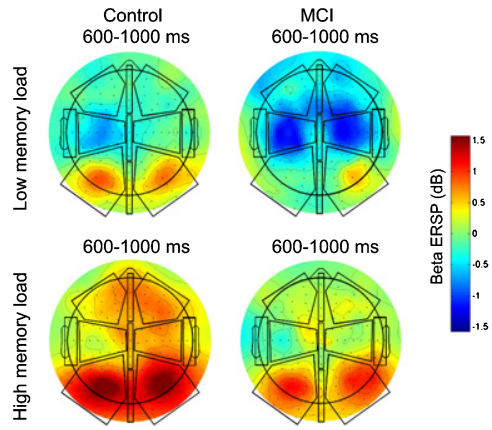

(B)

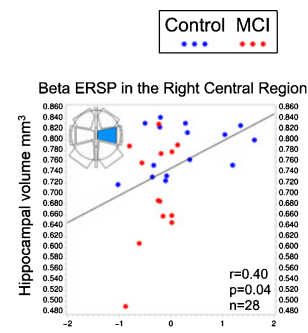

(C)

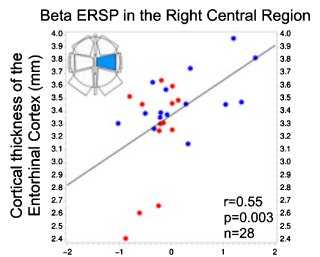

Fig. 4. Late retention condition. A) Beta event-related spectral perturbation (ERSP) in study groups (600-1000 ms time window highlighted). B) Scalp topography of the beta ERSP in study groups in the 600-1000 ms time window. C) Correlation of mean beta ERSP with the size of medial temporal lobe structures in the 600-1000 ms time window.

bition theory. According to this theory, increased alpha amplitude arising in cortical regions, that are not involved in stimulus processing protects the task-relevant information from interference [62]. Therefore decreased alpha ERS in MCI group might reflect deteriorated gating of task-irrelevant external information.

Correlation of alpha ERS with TMT results further support this assumption, as successful completion of TMT demands the effective filtering of interfering stimuli.

In late retention, alpha ERS did not show significant between-group difference. A potential reason for that might be that working memory retention is characterized by enhanced and attenuated alpha activity in different cortical regions simultaneously [21, 63, 64], and therefore during averaging these spectral changes might be smoothed out.

\section{Limitation}

The present study was limited by a slight age difference between groups, although statistical tests were corrected for age and gender as covariates and none of them had a significant effect in either condition. MRI data was not accessible for every subject as ten attendees refused participation. The study focused on the early diagnosis of cognitive impairment; however, involving patients with Alzheimer's disease or other type of dementia could paint a more detailed picture and expand the possible clinical application of the results. In this study, we did not apply a control task, which is a further limitation. Finally, followup data is not yet available to examine the predictive value of spectral perturbation in the conversion rate to dementia. However, our study provides a crosssectional view of the electrophysiological changes during working memory maintenance in MCI.

\section{Conclusion}

Working memory maintenance is accompanied by decreased alpha and beta ERS in MCI. While diminished ERS in the alpha frequency range during early retention might reflect deteriorated gating of external information in MCI patients, decreased beta ERS during early and late retention could mark the impairment of active information maintenance. In MCI, attenuated beta oscillatory activity in working memory maintenance network is observable during the early period of retention, primarily in the temporal lobe.

The assessment of electrophysiological changes especially in the beta frequency range may provide a useful diagnostic tool for the early detection of cog- 
nitive impairment, although further studies applying similar paradigms are required to verify our results.

\section{ACKNOWLEDGMENTS}

The study was supported by the 'Ambient Assisted Living Joint Programme (AAL) - Call 2' grant (G.C., project identifier AAL_08-1-2011$0005 \mathrm{M} 3 \mathrm{~W}$ ) (http://www.aaleurope.eu), by the grants from the Hungarian Research Fund (G.C., grant number OTKA PD 115837) and from the Semmelweis University (Zs.F., grant number EFOP3.6.3-VEKOP-16-2017-00009) and by the Bolyai Research Fellowship Program of the Hungarian Academy of Sciences (G.C.).

Authors' disclosures available online (https:// www.j-alz.com/manuscript-disclosures/17-1079r1).

\section{AVAILABILITY OF DATA AND MATERIAL}

The datasets used and/or analyzed during the current study are available from the corresponding author on reasonable request.

\section{ETHICAL STANDARDS}

The authors assert that all procedures contributing to this work comply with the ethical standards of the relevant national and institutional committees on human experimentation and with the Helsinki Declaration of 1975, as revised in 2008. The National Scientific and Ethical Committee, Budapest, Hungary approved the research. Participants gave their written informed consent before the procedures.

\section{SUPPLEMENTARY MATERIAL}

The supplementary material is available in the electronic version of this article: http://dx.doi. org/10.3233/JAD-171079.

\section{REFERENCES}

[1] Petersen RC (2004) Mild cognitive impairment as a diagnostic entity. J Intern Med 256, 183-194.

[2] Gauthier S, Reisberg B, Zaudig M, Petersen RC, Ritchie K, Broich K, Belleville S, Brodaty H, Bennett D, Chertkow H, Cummings JL, de Leon M, Feldman H, Ganguli M, Hampel H, Scheltens P, Tierney MC, Whitehouse P, Winblad $\mathrm{B}$, International Psychogeriatric Association Expert Conference on mild cognitive impairment (2006) Mild cognitive impairment. Lancet 367, 1262-1270.
[3] Csukly G, Sirály E, Fodor Z, Horváth A, Salacz P, Hidasi Z, Csibri É, Rudas G, Szabó Á (2016) The differentiation of amnestic type MCI from the non-amnestic types by structural MRI. Front Aging Neurosci 8, 52.

[4] Siraly E, Szabo A, Szita B, Kovacs V, Fodor Z, Marosi C, Salacz P, Hidasi Z, Maros V, Hanak P, Csibri E, Csukly G (2015) Monitoring the early signs of cognitive decline in elderly by computer games: An MRI study. PLoS One 10, e0117918.

[5] Deiber MP, Ibanez V, Missonnier P, Herrmann F, Fazio-Costa L, Gold G, Giannakopoulos P (2009) Abnormal-induced theta activity supports early directedattention network deficits in progressive MCI. Neurobiol Aging 30, 1444-1452.

[6] Deiber MP, Meziane HB, Hasler R, Rodriguez C, Toma S, Ackermann M, Herrmann F, Giannakopoulos P (2015) Attention and working memory-related EEG markers of subtle cognitive deterioration in healthy elderly individuals. J Alzheimers Dis 47, 335-349.

[7] Karrasch M, Laine M, Rinne JO, Rapinoja P, Sinerva E, Krause CM (2006) Brain oscillatory responses to an auditory-verbal working memory task in mild cognitive impairment and Alzheimer's disease. Int J Psychophysiol 59, 168-178.

[8] Missonnier P, Deiber MP, Gold G, Herrmann FR, Millet P, Michon A, Fazio-Costa L, Ibanez V, Giannakopoulos P (2007) Working memory load-related electroencephalographic parameters can differentiate progressive from stable mild cognitive impairment. Neuroscience 150, 346-356.

[9] Missonnier P, Gold G, Herrmann FR, Fazio-Costa L, Michel JP, Deiber MP, Michon A, Giannakopoulos P (2006) Decreased theta event-related synchronization during working memory activation is associated with progressive mild cognitive impairment. Dement Geriatr Cogn Disord 22, 250-259.

[10] Pijnenburg YA, v d Made Y, van Cappellen van Walsum AM, Knol DL, Scheltens P, Stam CJ (2004) EEG synchronization likelihood in mild cognitive impairment and Alzheimer's disease during a working memory task. Clin Neurophysiol 115, 1332-1339.

[11] Belleville S, Chertkow H, Gauthier S (2007) Working memory and control of attention in persons with Alzheimer's disease and mild cognitive impairment. Neuropsychology 21, 458-469.

[12] Brandt J, Aretouli E, Neijstrom E, Samek J, Manning K, Albert MS, Bandeen-Roche K (2009) Selectivity of executive function deficits in mild cognitive impairment. Neuropsychology 23, 607-618.

[13] Kirova AM, Bays RB, Lagalwar S (2015) Working memory and executive function decline across normal aging, mild cognitive impairment, and Alzheimer's disease. Biomed Res Int 2015, 748212.

[14] Saunders NL, Summers MJ (2011) Longitudinal deficits to attention, executive, and working memory in subtypes of mild cognitive impairment. Neuropsychology 25, 237-248.

[15] Summers MJ, Saunders NL (2012) Neuropsychological measures predict decline to Alzheimer's dementia from mild cognitive impairment. Neuropsychology 26, 498-508.

[16] Kensinger EA, Shearer DK, Locascio JJ, Growdon JH, Corkin S (2003) Working memory in mild Alzheimer's disease and early Parkinson's disease. Neuropsychology 17, 230-239.

[17] Gu L, Zhang Z (2017) Exploring potential electrophysiological biomarkers in mild cognitive impairment: A systematic 
review and meta-analysis of event-related potential studies. J Alzheimers Dis 58, 1283-1292.

[18] Makeig S (1993) Auditory event-related dynamics of the EEG spectrum and effects of exposure to tones. Electroencephalogr Clin Neurophysiol 86, 283-293.

[19] Busch NA, Herrmann CS (2003) Object-load and featureload modulate EEG in a short-term memory task. Neuroreport 14, 1721-1724.

[20] Leiberg S, Lutzenberger W, Kaiser J (2006) Effects of memory load on cortical oscillatory activity during auditory pattern working memory. Brain Res 1120, 131-140.

[21] Sauseng P, Klimesch W, Doppelmayr M, Pecherstorfer T, Freunberger R, Hanslmayr S (2005) EEG alpha synchronization and functional coupling during top-down processing in a working memory task. Hum Brain Mapp 26, 148-155.

[22] Jensen O, Gelfand J, Kounios J, Lisman JE (2002) Oscillations in the alpha band $(9-12 \mathrm{~Hz})$ increase with memory load during retention in a short-term memory task. Cereb Cortex 12, 877-882.

[23] Klimesch W (1999) EEG alpha and theta oscillations reflect cognitive and memory performance: A review and analysis. Brain Res Brain Res Rev 29, 169-195.

[24] Onton J, Delorme A, Makeig S (2005) Frontal midline EEG dynamics during working memory. Neuroimage 27, 341356.

[25] Tallon-Baudry C, Bertrand O, Peronnet F, Pernier J (1998) Induced gamma-band activity during the delay of a visual short-term memory task in humans. J Neurosci 18, 42444254.

[26] Chen Y, Huang X (2015) Modulation of alpha and beta oscillations during an n-back task with varying temporal memory load. Front Psychol 6, 2031.

[27] Schack B, Klimesch W (2002) Frequency characteristics of evoked and oscillatory electroencephalic activity in a human memory scanning task. Neurosci Lett 331, 107-110.

[28] Klimesch W (2012) Alpha-band oscillations, attention, and controlled access to stored information. Trends Cogn Sci 16, 606-617.

[29] Huang LY, She HC, Chou WC, Chuang MH, Duann JR, Jung TP (2013) Brain oscillation and connectivity during a chemistry visual working memory task. Int J Psychophysiol 90, 172-179.

[30] Palva S, Palva JM (2007) New vistas for alpha-frequency band oscillations. Trends Neurosci 30, 150-158.

[31] Deiber MP, Missonnier P, Bertrand O, Gold G, Fazio-Costa L, Ibanez V, Giannakopoulos P (2007) Distinction between perceptual and attentional processing in working memory tasks: A study of phase-locked and induced oscillatory brain dynamics. J Cogn Neurosci 19, 158-172.

[32] Missonnier P, Gold G, Herrmann FR, Fazio-Costa L, Michel JP, Deiber MP, Michon A, Giannakopoulos P (2006) Decreased theta event-related synchronization during working memory activation is associated with progressive mild cognitive impairment. Dement Geriatr Cogn Disord 22, 250-259.

[33] Li BY, Tang HD, Chen SD (2016) Retrieval deficiency in brain activity of working memory in amnesic mild cognitive impairment patients: A brain event-related potentials study. Front Aging Neurosci 8, 54.

[34] Tsolaki AC, Kosmidou V, Kompatsiaris IY, Papadaniil C, Hadjileontiadis L, Adam A, Tsolaki M (2017) Brain source localization of MMN and P300 ERPs in mild cognitive impairment and Alzheimer's disease: A high-density EEG approach. Neurobiol Aging 55, 190-201.
[35] Strauss E, Sherman E, Spreen O (2006) Compendium of Neuropsychological tests. Oxford University Press, pp. 168188.

[36] Sternberg S (1966) High-speed scanning in human memory. Science 153, 652-654.

[37] Barry RJ, Clarke AR, Johnstone SJ, Magee CA, Rushby JA (2007) EEG differences between eyes-closed and eyes-open resting conditions. Clin Neurophysiol 118, 2765-2773.

[38] Metting van Rijn AC, Peper A, Grimbergen CA (1990) High-quality recording of bioelectric events. Part 1. Interference reduction, theory and practice. Med Biol Eng Comput 28, 389-397.

[39] Delorme A, Makeig S (2004) EEGLAB: An open source toolbox for analysis of single-trial EEG dynamics including independent component analysis. J Neurosci Methods 134, 9-21.

[40] Mognon A, Jovicich J, Bruzzone L, Buiatti M (2011) ADJUST: An automatic EEG artifact detector based on the joint use of spatial and temporal features. Psychophysiology 48, 229-240.

[41] Makeig S, Debener S, Onton J, Delorme A (2004) Mining event-related brain dynamics. Trends Cogn Sci 8 , 204-210.

[42] Herrmann CS, Munk MH, Engel AK (2004) Cognitive functions of gamma-band activity: Memory match and utilization. Trends Cogn Sci 8, 347-355.

[43] Tallon-Baudry C, Bertrand O (1999) Oscillatory gamma activity in humans and its role in object representation. Trends Cogn Sci 3, 151-162.

[44] Pascual-Marqui RD (2002) Discrete, 3D distributed, linear imaging methods of electric neuronal activity. Part 1: Exact, zero error localization. http://arxiv.org/pdf/0710.3341

[45] Pascual-Marqui RD (2009) Theory of the EEG inverse problem. In Quantitative EEG Analysis: Methods and Clinical Applications, Tong S, Thankor NV, eds. Artech House, Boston, pp. 121-140.

[46] Hochberg Y (1988) A sharper Bonferroni procedure for multiple tests of significance. Biometrika 75, 800-802.

[47] Hochberg Y, Benjamini Y (1990) More powerful procedures for multiple significance testing. Stat Med 9, 811-818.

[48] Cohen J (1998) Statistical Power Analysis for the Behavioral Sciences., Lawrence Erlbaum Associates.

[49] Nichols TE, Holmes AP (2002) Nonparametric permutation tests for functional neuroimaging: A primer with examples. Hum Brain Mapp 15, 1-25.

[50] Gillis MM, Quinn KM, Phillips PA, Hampstead BM (2013) Impaired retention is responsible for temporal order memory deficits in mild cognitive impairment. Acta Psychol (Amst) 143, 88-95.

[51] Sano M, Raman R, Emond J, Thomas RG, Petersen R, Schneider LS, Aisen PS (2011) Adding delayed recall to the Alzheimer Disease Assessment Scale is useful in studies of mild cognitive impairment but not Alzheimer disease. Alzheimer Dis Assoc Disord 25, 122-127.

[52] Tierney MC, Szalai JP, Snow WG, Fisher RH, Nores A, Nadon G, Dunn E, St George-Hyslop PH (1996) Prediction of probable Alzheimer's disease in memory-impaired patients: A prospective longitudinal study. Neurology 46, 661-665.

[53] Hanslmayr S, Staresina BP, Bowman H (2016) Oscillations and episodic memory: Addressing the synchronization/desynchronization conundrum. Trends Neurosci 39, 16-25. 
[54] D'Esposito M (2007) From cognitive to neural models of working memory. Philos Trans R Soc Lond B Biol Sci 362, 761-772.

[55] Nee DE, D'Esposito M (2018) The representational basis of working memory. Curr Top Behav Neurosci 37, 213-230.

[56] Ranganath C (2006) Working memory for visual objects: Complementary roles of inferior temporal, medial temporal, and prefrontal cortex. Neuroscience 139, 277-289.

[57] Campo P, Poch C (2012) Neocortical-hippocampal dynamics of working memory in healthy and diseased brain states based on functional connectivity. Front Hum Neurosci 6, 36.

[58] Axmacher N, Schmitz DP, Wagner T, Elger CE, Fell J (2008) Interactions between medial temporal lobe, prefrontal cortex, and inferior temporal regions during visual working memory: A combined intracranial EEG and functional magnetic resonance imaging study. $J$ Neurosci $\mathbf{2 8}$, 7304-7312.

[59] Prieto del Val L, Cantero JL, Atienza M (2015) APOE varepsilon4 constrains engagement of encoding-related compensatory networks in amnestic mild cognitive impairment. Hippocampus 25, 993-1007.
[60] Aurtenetxe S, Castellanos NP, Moratti S, Bajo R, Gil P, Beitia G, del-Pozo F, Maestu F (2013) Dysfunctional and compensatory duality in mild cognitive impairment during a continuous recognition memory task. Int J Psychophysiol 87, 95-102.

[61] Giannakopoulos P, Missonnier P, Kovari E, Gold G, Michon A (2009) Electrophysiological markers of rapid cognitive decline in mild cognitive impairment. Front Neurol Neurosci 24, 39-46.

[62] Jensen O, Mazaheri A (2010) Shaping functional architecture by oscillatory alpha activity: Gating by inhibition. Front Hum Neurosci 4, 186.

[63] Klimesch W, Sauseng P, Hanslmayr S (2007) EEG alpha oscillations: The inhibition-timing hypothesis. Brain Res Rev 53, 63-88.

[64] Meltzer JA, Zaveri HP, Goncharova II, Distasio MM, Papademetris X, Spencer SS, Spencer DD, Constable RT (2008) Effects of working memory load on oscillatory power in human intracranial EEG. Cereb Cortex 18, 1843-1855. 